\title{
Contamination by field late-M, L, and T dwarfs in deep surveys
}

\author{
J. A. Caballero ${ }^{1,2}$, A. J. Burgasser ${ }^{3}$, and R. Klement ${ }^{1}$ \\ 1 Max-Planck-Institut für Astronomie, Königstuhl 17, 69117 Heidelberg, Germany \\ e-mail: caballero@astrax.fis.ucm.es \\ 2 Dpto. de Astrofísica y Ciencias de la Atmósfera, Facultad de Ciencias Físicas, Universidad Complutense de Madrid, 28040 Madrid, \\ Spain \\ 3 Massachusetts Institute of Technology, Kavli Institute for Astrophysics and Space Research, Cambridge, MA 02139, USA
}

Received 6 February 2008 / Accepted 26 May 2008

\section{ABSTRACT}

\begin{abstract}
Context. Deep photometric surveys of substellar objects in young clusters and high-redshift quasars are affected by contaminant sources at different heliocentric distances. If not correctly taken into account, the contamination may have a strong effect on the Initial Mass Function determination and on the identification of quasars.

Aims. We calculate in detail the back- and foreground contamination by field dwarfs of very late spectral types (intermediate and late $\mathrm{M}, \mathrm{L}$, and $\mathrm{T}$ ) in deep surveys and provide the data and tools for the computation.

Methods. We adopt the latest models and data from the literature, which include the following: $(i)$ a model of the Galactic thin disc by an exponential law; (ii) the length and height scales of late-type dwarfs; and (iii) the local spatial densities, absolute magnitudes, and colours of dwarfs for each spectral type.

Results. We derive a simplified expression for the spatial density in the thin disc that depends on the heliocentric distance and the galactic coordinates $(l, b)$ and integrate this into the truncated cone studied in the survey. As a practical application, we compute the numbers of $\mathrm{L}$ - and T-type field dwarfs in very deep $(I=21-29 \mathrm{mag})$ surveys in the direction of the young $\sigma$ Orionis cluster. The increasing number of contaminants at the faintest magnitudes could inhibit the study of the opacity mass limit at $M \lessgtr 0.003 M_{\odot}$ in the cluster.
\end{abstract}

Key words. stars: low-mass, brown dwarfs - stars: luminosity function, mass function - Galaxy: stellar content Galaxy: open clusters and associations: individual: $\sigma$ Orionis - methods: analytical

\section{Introduction}

Deep wide-field multi-band photometric searches in open clusters and star-forming regions are the most useful tool for the discovery and identification of young brown dwarfs and planetarymass objects (e.g. Rebolo et al. 1996; and Bouvier et al. 1998, in the Pleiades; Preibisch \& Zinnecker 1999 and Ardila et al. 2000, in Upper Scorpius; Zapatero Osorio et al. 2000 and Béjar et al. 2001, in $\sigma$ Orionis). Unfortunately, an important fraction of the selected cluster member candidates do not belong to the cluster, but are interlopers in the fore- and background. This fact strongly affects posterior analyses of the bottom of the Initial Mass Function, down to about a few Jupiter masses. There are several ways of reducing the contamination of the mass function incurred by these interlopers, including the identification of spectral indicators of youth/low surface gravity in individual sources (Martín et al. 1996; Stauffer et al. 1998; White \& Basri 2003; McGovern et al. 2004; Mohanty et al. 2005) and removing sources with kinematics inconsistent with the cluster (Stauffer et al. 1994; Hambly et al. 1999; Moraux et al. 2001; Bihain et al. 2006; Lodieu et al. 2007b). However, these techniques may require prohibitive investments of time on large telescopes, particularly for the lowest mass objects (e.g. S Ori 70 - see below), and may not eliminate contaminants for distant and/or older clusters and associations. For example, at $100 \mathrm{pc}$, accuracies of higher quality than $\sim 10 \mathrm{mas} / \mathrm{a}^{-1}$ are required to measure velocities of $\sim 5 \mathrm{~km} \mathrm{~s}^{-1}$. In that case, there can still be foreground objects that share the same motions but are not cluster members. Their membership status can only be ensured by the detection of youth signatures. Some known features of extreme youth (age $\lesssim 10 \mathrm{Ma}$ ) are Li I $26707.8 \AA$ in absorption (Rebolo et al. 1992), abnormal strength of alkali lines and water and $\mathrm{H}_{2}$ absorption bands due to low gravity (Martín et al. 1999; Kirkpatrick et al. 2006; Allers et al. $2007-\mathrm{H}_{2}$ is also detected in emission from discs; Thi et al. 2001), strong $\mathrm{H} \alpha \lambda 6562.8 \AA$ emission associated with accretion, and mid-infrared flux excesses due to circum(sub)stellar discs. However, acquiring high signal-to-noise ratio mid-resolution spectroscopy or Spitzer Space Telescope imaging of the faintest, reddest objects in a deep photometric survey $(I \gtrsim 18-19 \mathrm{mag}$, $J \gtrsim 15-16 \mathrm{mag}$ ) can be an extremely difficult task.

A useful statistical approach is to measure both the luminosity function in the area of the cluster and in neighbouring fields, and extract the true cluster luminosity function as the difference of these. This method was applied successfully to the Orion Nebula Cluster and described in detail by for instance Muench et al. (2002). Besides relying on a local extinction map of the cluster to redden the stellar population in extinction-free comparison fields, the method, regrettably, has one important handicap: we have to integrate for the same exposure time in the neighbouring fields to properly subtract the "field mass function". For the deepest surveys in clusters, using expensive large facilities, this time-consuming solution cannot be accomplished in many cases. As a result, the vast majority of these deep surveys establish certain selection criteria for cluster membership based on colour-magnitude diagrams.

Depending on the criteria and the number and suitability of passbands, more or less cluster non-member contaminants 
could be among the selected member candidates in a photometric survey. To define a selection criterion, the authors must account for photometric uncertainties in the survey, the natural scatter of the cluster sequence (i.e. the sources themselves do not have a single absolute magnitude or colour, but rather a range of both appropriate for their spectral type), unresolved binarity, and intrinsic photometric variability. Figure 2 in Caballero et al. (2007) illustrates a typical selection of member candidates in the $\sigma$ Orionis cluster based on the loci of spectroscopically confirmed members in an $I$ versus $I-J$ diagram. Theoretical isochrones may be used as reference, as well. The removal of interlopers, whose location coincides with that of cluster members in colour-magnitude diagrams, is important in the faintest and reddest magnitude intervals. Quantitative estimations of contaminants were presented in several searches in young clusters (Paresce et al. 1995; Zapatero Osorio et al. 1997; Martín et al. 2000; Lucas et al. 2001; Jeffries et al. 2004; Moraux et al. 2007).

In this paper, we provide the necessary tools and state-of-theart data for the correct decontamination of field late-type stars and brown dwarfs in deep photometric surveys at intermediate and high galactic latitudes. A practical application of the integrated number of contaminants in very deep surveys towards the $\sigma$ Orionis cluster is presented. Our work can be applied not only to searches in other open clusters and star-forming regions, but also to high-z quasar surveys.

\section{Analysis}

\subsection{Possible contaminants}

The contaminants appearing in deep photometric searches for very red objects are in general faint reddened galaxies, variable subgiants, (reddened) distant giants, or field dwarfs with very late spectral types that fall close to the sequence of the cluster under study in a colour-magnitude diagram:

- galaxies are in general extended sources, so their FWHMs are larger than the average FWHM of stars in a particular field of view, and they can easily be rejected by the point-spread-function photometry: down to $J \sim 19-20 \mathrm{mag}$, galaxies can be detected under good seeing conditions (see, for example, Foster et al. 2008). They also display colours that do not match the dwarf sequence in a colour-colour diagram (e.g. $J-K_{\mathrm{s}}$ versus $I-J$ ). Some remote quasars with $z \sim 6$ have, however, point-like FWHMs and spectral energy distributions almost identical to those of mid-T dwarfs; these quasars are, nevertheless, extremely rare (Stern et al. 2007);

- apart from extremely young star forming regions with high extinction clouds ( $\rho$ Ophiuchi, Chamaeleon I, Orion Nebula Cluster), open clusters are relatively free of interstellar absorption. In some clusters, such as $\sigma$ Orionis, the strong wind and ultraviolet radiation from the Trapezium-like central system has completely cleared the region from cometary globulae, molecular clouds, and most of the original intracluster material. In the case of clusters with thick molecular clouds, these can act as a "shield to background field stars" (Lucas \& Roche 2000; Luhman et al. 2000; Muench et al. 2002), which may facilitate the decontamination (there will be only interloping non-cluster field stars in the foreground);

- according to Kirkpatrick et al. (1994), contamination by giant stars in areas excluding the Galactic plane is negligible. Given the absolute magnitude of late-type giant stars, a hypothetical faint (intrinsically bright) giant contaminant would be at several kiloparsecs over or below the Galactic plane, where only the globular clusters are found. A comparatively large number of giant interlopers have been identified in some studies. For example, Cruz et al. (2007) identified 33 giants and 17 carbon stars among a list of 200 ultracool dwarf candidates in the field. However, they only used 2MASS $J H K_{\mathrm{s}}$ photometry for the selection. The employment of at least one optical filter and astrometric and spectroscopic information from the literature can help to eliminate these contaminants in cluster (and field) surveys;

- photometric variability of red (sub-)giants in the background is an additional source of contamination for surveys whose multiband images were taken at different epochs (i.e. the colours of variable sources are not "real"). Many of these contaminants, although difficult to discard a priori, can be identified by studying their light curves. The deep final image in a passband of a survey is often the combination of a long series of exposures; this methodology, if accompanied by suitable dithering, enables deeper magnitudes to be detected than by a single exposure in the same total time. See Caballero et al. (2004) and Scholz \& Eislöffel (2004) for examples of photometric variability studies in a young open cluster based on such a long series of observations;

- the major contributors to red contamination in deep surveys of clusters are, therefore, field very low-mass stars and brown dwarfs of late spectral types. The discovery of distant, late-type dwarfs in deep surveys is unexceptional. For example, Stanway et al. (2008) found a population of $\mathrm{M}$ dwarfs at large heliocentric distances in the GOODS fields. Pirzkal et al. (2005) also identified $18 \mathrm{M}$ and two L-type candidate dwarfs in the Hubble Ultra Deep Field, which were confirmed following. Many more field $\mathrm{L}$ dwarfs have been found in the foregrounds of young open clusters and associations (e.g. $\sigma$ Orionis: Caballero et al. 2007; Upper Scorpius: Lodieu et al. 2008). During an extragalactic survey, Cuby et al. (1999) identified the most distant field T dwarf to date (NTTDF J1205-0744), at a heliocentric distance of $d \sim 90 \mathrm{pc}$ and with a magnitude of $J \sim 20$ mag. Kendall et al. (2007) and Lodieu et al. (2007a) estimated slightly lower distances $(d \sim 50-80)$ for the intermediate T dwarfs ULAS J145243.59+065542.9 and ULAS J223955.76+003252.6, respectively. The precise status of SOri 70, a $\sim$ T6-type, $\sim 3 M_{\text {Jup }}$, planetarymass object candidate towards Orion, is still under debate (Zapatero Osorio et al. 2002b, 2008; Martín \& Zapatero Osorio 2003; Burgasser et al. 2004; Scholz \& Jayawardhana 2008).

\subsection{The Galaxy thin-disc model}

Within the context of a "standard" Galactic model, the Galaxy can be modelled by a double exponential, representing the thin and the thick discs, and a power law for the halo (e.g. a de Vaucouleurs-type power law). Classic works and reviews on Galactic structure were presented by, e.g. Bahcall \& Soneira (1980), Gilmore \& Reid (1983), Majewski (1993), and Kroupa et al. (1993). Analyses of deep imaging survey data using Galaxy disc models were completed by, for example, Phleps et al. (2005), Ryan et al. (2005), Karaali (2006), and Jurić et al. (2008).

The volume densities of the axisymmetric thin and thick discs depend on the galactocentric distance $R$ and the height over or below the galactic plane, $Z$. For an object of spectral type $i$, the expression for its spatial density depending on heliocentric 
galactic coordinates $(l, b)$ and heliocentric distance $(d)$ is usually written as follows:

$n_{i}(d, l, b)=n_{0, i} \mathrm{e}^{-\frac{R(d, l, b)-R_{\odot}}{h_{R}}} \mathrm{e}^{-\frac{\left|Z_{\odot}+d \sin b\right|}{h_{Z}}}$

(e.g. Chen et al. 2001, and references therein), where $n_{0, i}$ is the star spatial density at the Galactic plane $(Z=0)$ and at the solar galactocentric distance $\left(R=R_{\odot}\right), Z_{\odot}$ is the height of the Sun over the Galactic plane, and $h_{R}$ and $h_{Z}$ are the length (radial) and height scales, respectively. The exponential vertical distribution originates in the approximation $\operatorname{sech}^{2}\left(|Z| / 2 h_{Z}\right) \approx \mathrm{e}^{-|Z| / h_{Z}}$ (Gilmore et al. 1990). We repudiate using more complex forms for the vertical density profile (e.g. Zheng et al. 2004), since additional parameters that characterize these forms (e.g. $\beta$ ) are more poorly constrained than the exponential scale height factors (see also the following discussion on the thin-to-thick disc normalisation). The galactocentric distance $R(d, l, b)$ of the object is then:

$R(d, l, b)=\left(R_{\odot}^{2}+d^{2} \cos ^{2} b-2 R_{\odot} d \cos b \cos l\right)^{1 / 2}$

(e.g. Bahcall \& Soneira 1980, where $R$ is measured along the Galactic plane). By assuming that the heliocentric distance, $d$, is far lower than the solar galactocentric distance, $d \ll R_{\odot}$ (see below for a justification), then the galactocentric distance of the object can be approximated by:

$R(d, l, b) \approx R_{\odot}-d \cos b \cos l$.

The minus sign guarantees that $R<R_{\odot}$ when the line of sight points to the Galactic Centre $(l=0 \mathrm{deg})$, and vice versa, where we use the right-handed Cartesian galactocentic coordinate system in which the $X$ axis points towards the Sun, the $Y$ axis in the anti-direction of Galactic rotation, and the $Z$ axis towards the North Galactic Pole. In the linear approximation, Eq. (1) can be rewritten as:

$n_{i}(d, l, b) \approx n_{0, i} \mathrm{e}^{\frac{d \cos b \cos l}{h_{R}}} \mathrm{e}^{-\frac{ \pm Z_{\odot} \pm d \sin b}{h_{Z}}}=n_{0, i} \mathrm{e}^{\frac{\mp Z_{\odot}}{h_{z}}} \mathrm{e}^{-d\left(-\frac{\cos b \cos l}{h_{R}} \pm \frac{\sin b}{h_{Z}}\right)}$,

where the sign convention depends on whether $d \sin b$ is greater or less than $-Z_{\odot}$ (i.e. whether the source is above or below the Galactic plane). Defining two auxiliar variables that vary with the galactic coordinates, $n_{A, i}$ and $d_{B}(l, b)$, Eq. (1) produces a simple expression in terms of the heliocentric distance:

$n_{i}(d, l, b) \approx n_{A, i} \mathrm{e}^{-\frac{d}{d^{(l, b)}}}$,

where the auxiliar variables are computed by:

$n_{A, i} \equiv n_{0, i} \mathrm{e}^{\mp \frac{Z_{\odot}}{h_{Z}}}$

and

$\frac{1}{d_{B}(l, b)} \equiv-\frac{\cos b \cos l}{h_{R}} \pm \frac{\sin b}{h_{Z}}$.

In Table 1, we indicate the main parameters of the Galaxy thin-disc model from Chen et al. (2001). These values fit suitably classic and recent determinations (e.g. Faber et al. 1976; Bahcall \& Soneira 1984; Kerr \& Lynden-Bell 1986; Kuijken \& Gilmore 1989; Robin et al. 2000; Pirzkal et al. 2005; Jurić et al. 2008, and references above). We use subsequently only Chen et al.'s values for consistency, even though more accurate estimates of some parameters exist (e.g. the solar galactocentric distance - Reid 1993; Eisenhauer et al. 2003). From the constancy of the height scale of GKM-type dwarfs as shown by Bahcall \& Soneira (1980) and Gilmore \& Reid (1983), it appears
Table 1. Main parameters of the Galaxy thin-disc model from Chen et al. (2001).

\begin{tabular}{lcccc}
\hline \hline Parameter & $R_{\odot}$ & $Z_{\odot}$ & $h_{R}$ & $h_{Z}$ \\
& $(\mathrm{pc})$ & $(\mathrm{pc})$ & $(\mathrm{pc})$ & $(\mathrm{pc})$ \\
\hline Value & $8600 \pm 200$ & $+27 \pm 4$ & $2250 \pm 1000$ & $330 \pm 3$ \\
\hline
\end{tabular}

reasonable to use the same height scale for $\mathrm{L}$, and $\mathrm{T}$ dwarfs. Pirzkal et al. (2005) measured $h_{z}=400 \pm 100 \mathrm{pc}$ for late M and L dwarfs, which is slightly higher than our measurement, but still consistent. While the vertical scale heights of early-type stars $(\mathrm{O}, \mathrm{B}, \mathrm{A})$ are smaller, they do not contribute significantly to the photometrically selected sample and are in all cases rare.

The thick disc is more rarified and extended than the thin disc with a thin-to-thick disc normalisation factor in the solar vicinity of 13-6.5\% (Chen et al. 2001; Jurić et al. 2008). This range of measured values is wider than reported before, such as $2 \%$ by Gilmore (1984) or the lack of evidence for a thick disc in Bahcall \& Soneira (1984). According to theoretical cooling sequences (Burrows et al. 1997; Chabrier et al. 2000; Baraffe et al. 2003), very old thick-disc substellar objects with very low masses $\left(\mathcal{M} \ll 0.08 M_{\odot}\right)$ may be dimmed to exceedingly faint magnitudes, which would prevent their detection (thick-disc stars and brown dwarfs are thought to be very old, with ages $\gtrsim 10 \mathrm{Ga}-$ e.g. Fuhrmann 1998; Prochaska et al. 2000; Feltzing et al. 2003). For example, a 1 Ga-old, $0.05 M_{\odot}$-mass brown dwarf has an effective temperature typical of an L5-7V dwarf and an absolute magnitude of $M_{I}=17.2 \mathrm{mag}$; nine gigayears later (i.e. age $=10 \mathrm{Ga}$ ), the brown dwarf will dim to $M_{I}=20.5 \mathrm{mag}$ and cool down to T7-9V spectral type. Since very low-mass stars above the hydrogen burning limit (with late $\mathrm{M}$ and very early $\mathrm{L}$ spectral types) maintain an approximately constant luminosity in the main sequence for tens of gigayears, we expect that the ratio of the number of L dwarfs to that of T (and cooler) dwarfs in the (relatively old) thick disc is lower than in the (relatively young) thin disc. In other words, field late-M-, L- and T-type dwarfs of the solar neighbourhood are younger, on average, than G-, K- and early M-type dwarfs (see Table 5 in Zapatero Osorio et al. 2007a). There is discussion about the negligibility of thick disk and halo brown dwarfs in a given imaging sample in Pirzkal et al. (2005). Both the low thin-to-thick disc normalisation factor and the relative faintness of thick-disc objects suggest that it is appropriate to use just the thin-disc exponential for determining the density of late-type dwarfs in the Galaxy. The error in this assumption $(\lesssim 10 \%)$ is smaller than, or of the order of, the uncertainties in the determination of $R_{\odot}, Z_{\odot}$, and $h_{R}$.

Using the values in Table 1 , the ratio $n_{A, i} / n_{0, i}=\mathrm{e}^{\mp \frac{Z_{\odot}}{h_{Z}}}$ (Eq. (6)) equals $0.921 \pm 0.011$ for $Z>0$, and $1.085 \pm 0.013$ for $Z<0$ and is independent of the galactic coordinates. However, the auxiliary variable $d_{B}(l, b)$ depends strongly on the galactic longitude and latitude in the direction of observation and has a large interval of variation.

Figure 1 shows the suitability and accuracy of the linear approximation of $R(d, l, b)$. The differences between the relative spatial densities of stars and brown dwarfs of spectral type $i$ in the thin disc, $n(d) / n_{0}$, using the correct (Eq. (2)) and approximate (Eq. (3)) expressions are no larger than $0.2 \%$ in the direction of three representative open clusters, whose galactic coordinates and distances are given in Table 2. The differences are two orders of magnitude lower $(<0.002 \%)$ at heliocentric distances $d<100 \mathrm{pc}$, where the expected number of late-type dwarf contaminants reaches a maximum. These differences are very small in comparison with the errors in the determination of the 

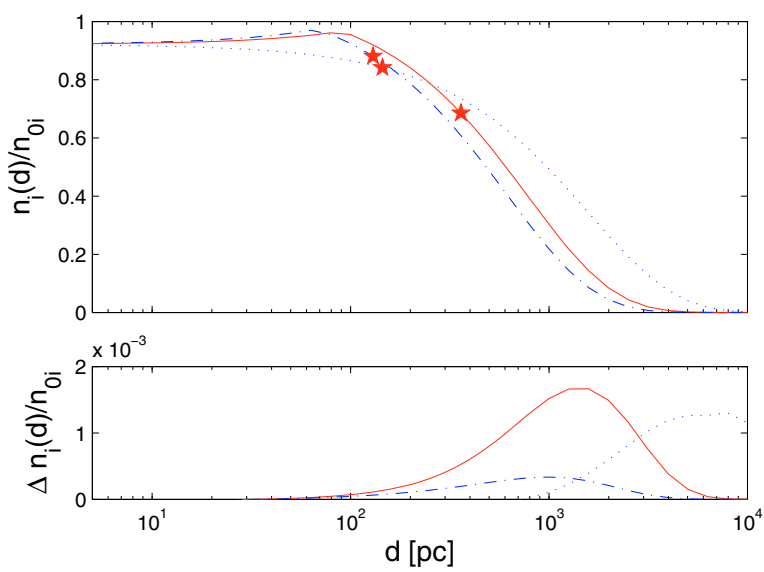

Fig. 1. Top window: variation in the relative spatial density of stars and brown dwarfs of spectral type $i$ in the thin disc with the heliocentric distance from 5 to $10000 \mathrm{pc}$ in the direction of three representative young clusters: Upper Scorpius (dotted line), Pleiades (dash-dotted line), and $\sigma$ Orionis ( - red - solid line). The location of the clusters are indicated by a star " $\star$ " symbol. Bottom window: differences between the relative spatial densities using the correct (Eq. (2)) and approximate (Eq. (3)) expressions.

Table 2. Heliocentric distances and galactic coordinates of three representative young open clusters.

\begin{tabular}{lccc}
\hline \hline Region & $\begin{array}{c}d \\
(\mathrm{pc})\end{array}$ & $\begin{array}{c}l \\
(\mathrm{deg})\end{array}$ & $\begin{array}{c}b \\
(\mathrm{deg})\end{array}$ \\
\hline Upper Scorpius & 145 & 350 & +20 \\
$\sigma$ Orionis & 385 & 207 & -17 \\
Pleiades & 130 & 167 & -24 \\
\hline
\end{tabular}

main parameters of the Galaxy disc model and, as we will see later, the spatial densities of each spectral type. Besides, the linear $R(d, l, b)$ approximation simplifies enormously the following analysis.

All $n(d) / n_{0}$ curves in the top panel of Fig. 1 show the same local density at null heliocentric distance, which is for an object of spectral type $i$ :

$n_{i}\left(R=R_{\odot}, Z=Z_{\odot}\right)=n_{0, i} \mathrm{e}^{-\frac{Z_{\odot}}{h_{z}}} \equiv n_{\odot, i}$.

Upper Scorpius is in the Galactic northern hemisphere, like the Sun, so the line of sight of the search does not cross the Galactic plane. Thus, in contrast to the Pleiades and $\sigma$ Orionis, the Upper Scorpius relative star density does not reach a maximum at $Z=0$ (and $n_{A, i}(d, b)$ and $d_{B, i}(d, l, b)$ do not change sign). For the Pleiades and $\sigma$ Orionis, the peak of $n(d) / n_{0}$ at $d \sin b=-Z_{\odot}$ (when the line of sight intersects the Galactic plane) is not exactly 1.00 , but there is an error of about $3 \%$ because the galactic coordinate system "is necessarily based on a Galactic plane through the Sun", which is the H I principal plane (Blaauw et al. 1960). Finally, the spatial density in the direction to Upper Scorpius is lower than in the direction to the other clusters at relatively small heliocentric distances, but it becomes higher beyond about $250 \mathrm{pc}$ because the $\mathrm{OB}$ association is approximately in the direction of the Galactic Centre.

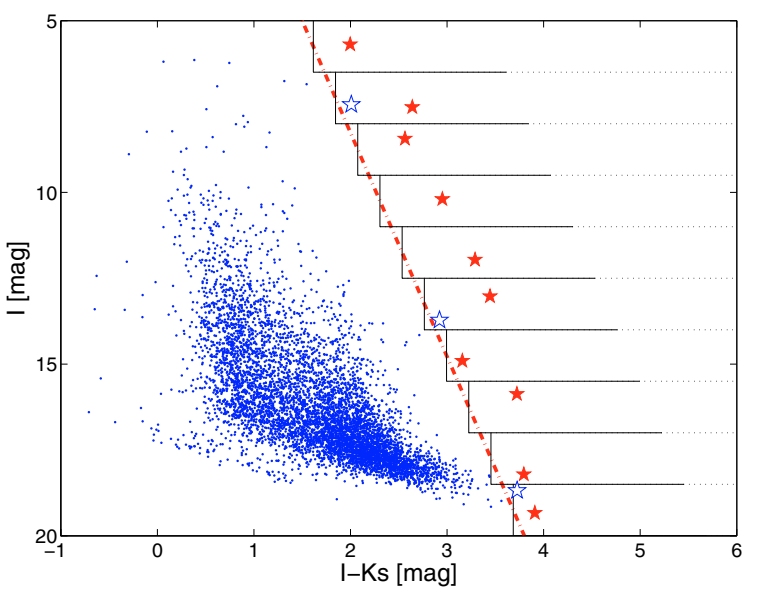

Fig. 2. Simulated $I_{\mathrm{SSA}}$ vs. $I_{\mathrm{SSA}}-K_{\mathrm{s}, 2 \mathrm{MASS}}$ colour-magnitude diagram of a hypothetical cluster survey. (Red) Filled stars (10): hypothetical confirmed cluster members with spectroscopic signatures of youth; (blue) open stars (3): hypothetical cluster member candidates based on photometry; (blue) small dots: probable fore- and background sources; (red) thick dot-dashed line: selection criterion used for separating cluster members and candidates from probable fore- and background sources; (black) thin solid/dotted lines: "strips" where the number of contaminants are computed. The height of the strips were set to $1.5 \mathrm{mag}$ for clarity. Small dots and open stars are actual data from a multiband $\left(B R I_{\mathrm{SSA}} J H K_{\mathrm{s}, 2 \mathrm{MASS}}\right) 90$ arcmin-radius survey centred on HD 221356 (aka Kö $3 \mathrm{~A}$ ), an F8V star with an M-L-type binary companion in a very wide orbit (Gizis et al. 2000; Caballero 2007). The mean sequence of the hypothetical cluster is parallel to the thick dot-dashed line, shifted $0.5 \mathrm{mag}$ to the red. Filled stars were generated randomly by adding a 0.5 mag-amplitude noise to the mean cluster sequence (i.e. the selection criterion is $\sim 1 \sigma$ shifted to the blue with respect to the mean cluster sequence). (SSA: SuperCOSMOS Science Archive, Hambly et al. 2001; 2MASS: Two-Micron All Sky Survey, Skrutskie et al. 2006).

\subsection{Integrated number of contaminants}

\subsubsection{The pseudo-algorithm}

As introduced in Sect. 1, the number of possible field-dwarf contaminants in a survey can be computed from the corresponding colour-magnitude diagram. In Fig. 2, we illustrate the computation of contaminants based on a simulated colour-magnitude diagram. The pseudo-algorithm of integration is identical for any combination of "red" (e.g. $K_{\mathrm{s}}$ ) and "blue" (e.g. $I$ ) passbands.

The general procedure to compute the integrated number of field-dwarf interlopers consists of:

1. Defining horizontal "strips" in the colour-magnitude diagram. These are discrete rectangular regions in the diagram that divide the area of selected sources into pieces for a suitable integration. The blue border of each strip is defined by the selection criterion; the choice of the red border is, however, subjective, because observers tend to select cluster member candidates to be all the sources to the red of the selection criterion cut off. A reasonable choice of red border is the colour of the reddest source in each magnitude interval, but it can be extended up to very red colours (indicated by dotted lines in Fig. 2). The height of the strips (i.e. the size of the integration step) depends on the required accuracy; a width of $\sim 0.5$ mag can be a compromise between a rapid computation and fidelity in the reproduction of the shape of the selection criterion (in this case, a straight line). This height cannot be smaller than the typical photometric uncertainty. 
2. Determining the spectral types of the possible interlopers in each strip. They are derived from the colours at the strip borders and from a spectral type-colour relation. See below for caveats of this determination.

3a. Setting the minimum and maximum heliocentric distances $d_{1}$ and $d_{2}$, at which an interloper of a given spectral type would occupy each strip, based on the corresponding absolute magnitudes, $M_{\lambda}$. The computation of $M_{\lambda}$ must account for the apparent magnitude $m_{\lambda}$, the heliocentric distance $d$, and the interstellar extinction $A_{\lambda}$ (the error in the $M_{\lambda}$-spectral type relation is considered in item \#2).

3b. Setting the heliocentric distance $d_{*}$ at which the line of sight crosses the Galactic plane, in case the minimum and maximum heliocentric distances are in different Galactic hemispheres.

4. Integrating the spatial density between the minimum and maximum heliocentric distances to derive the number of interlopers of a given spectral type per surface unit in each strip.

5. Summing the number of interlopers, of all the considered spectral types and in all the strips.

6. Multiplying the value by the survey area, $\mathcal{S}$, to calculate the integrated number of contaminants.

Regarding item \#2, a field dwarf with intrinsic colour bluer than the selection limit can occupy the selected region of the colour-magnitude diagram because of photometric error. Moreover, a spectral type does not correspond to a single absolute magnitude and one colour value but to a range of magnitudes and colours. It is necessary to take these effects into account when calculating the contamination, otherwise the result will be underestimated. One means of accounting for the photometric scattering of the data (including the broadening of the main sequence and the survey photometric uncertainties) may be selecting an earlier spectral subtype of bluer interloper than expected from the selection criterion. At the considered late spectral types, a variation of one subtype corresponds to an average colour variation of, e.g. $\Delta(I-J) \approx 0.2-0.3$ mag (see below), which may be a too large photometric uncertainty for a typical survey. An alternative solution is to shift bluewards the selection criterion used in the colour-magnitude diagram by $\sim 0.05-0.10 \mathrm{mag}$. The precise amount would depend on the survey, magnitude range, and considered spectral types.

The integration can be completed when the main parameters (Table 1) and the exponential form of the Galaxy thin-disc model (Eq. (1)) are known, as well as the spatial densities, colours, and absolute magnitudes of the possible contaminants as a function of spectral type (Sect. 2.4).

\subsubsection{The integration}

We describe the tools required to integrate the exponential spatial density using the linear approximation (Eq. (5)). In a typical photometric search program, the search volume can be approximated by a cone of apex length that is equal to the heliocentric distance of a source detectable above the photometric depth, and a base corresponding to the projected area on the sky of the search, $\mathcal{S}$. We assume that the search is not over an excessively wide area, that is it is smaller than a few tens of square degrees. The axis of the cone is defined by the line of sight (i.e. the galactic latitude and longitude of the centre of the survey area). The use of a cone simplifies the computation of late-type contaminants, since it is not necessary to know the precise shape of the survey.
The number of late-type dwarfs of spectral type $i$ that contaminate the survey is calculated by the integration in a truncated cone of volume $V$ with a non-uniform density $n(\boldsymbol{r})$ :

$N_{i}=\int n_{i}(d) \mathrm{d} V=\int_{z} \int_{\phi} \int_{\alpha} n_{i}(z, \phi, \alpha) \mathrm{d} V$,

where the volume differential in the coordinate system of the cone is $\frac{z^{2} \sin \alpha}{\cos ^{3} \alpha} \mathrm{d} z \mathrm{~d} \phi \mathrm{d} \alpha$ (where $z$ is the conventional $z$-coordinate, $\phi$ is the angle of a projected vector with the positive $X$-axis, measured counterclockwise, and $\alpha$ is the angle between the vertical axis, the cone vertex, and any other point of the cone). When the galactic latitude and longitude of the field of view are known, the density $n(\boldsymbol{r})$ depends only on the heliocentric distance, $d$ (the "height" of the cone). Using the simplified expression for the density of thin-disc dwarfs (Eq. (5)), the integration between the heliocentric distances $d_{1}$ and $d_{2}$ provides:

$$
\begin{aligned}
N_{i} & =\int_{d_{1}}^{d_{2}} n_{i}(z) z^{2} \mathrm{~d} z \int_{0}^{2 \pi} \mathrm{d} \phi \int_{0}^{\arctan (b / h)} \frac{\sin \alpha}{\cos ^{3} \alpha} \mathrm{d} \alpha \\
& =\pi\left(\frac{b}{h}\right)^{2} \int_{d_{1}}^{d_{2}} n_{A, i} \mathrm{e}^{-\frac{z}{d_{B}}} z^{2} \mathrm{~d} z
\end{aligned}
$$

In the pyramid-cone approximation of height $h$ and base radius $b$, the value $\pi\left(\frac{b}{h}\right)^{2}$ is simply the angular area of the search, $\mathcal{S}$, which must be expressed in $\operatorname{rad}^{2}$. If $d_{1}$ and $d_{2}$ are each in a Galactic hemisphere (i.e. the line of sight crosses the Galactic plane), then the integral is detached into two parts:

$N_{i}=\mathcal{S}\left(n_{A_{+, i}} \int_{d_{1}}^{d_{*}} \mathrm{e}^{-\frac{z}{d_{B_{+}}}} z^{2} \mathrm{~d} z+n_{A_{-, i}} \int_{d_{*}}^{d_{2}} \mathrm{e}^{-\frac{z}{d_{B-}}} z^{2} \mathrm{~d} z\right)$,

where $d_{*}$ is the heliocentric distance at which $n(d)$ has a maximum $(Z=0)$ and the $n_{A}$ and $d_{B}$ auxiliary variables change behaviour. The exponential integral has a primitive, which is:

$$
\begin{aligned}
\int_{d_{i}}^{d_{j}} \mathrm{e}^{-\frac{z}{d_{B}}} z^{2} \mathrm{~d} z & =\left[(-1) \mathrm{e}^{-\frac{z}{d_{B}}}\left(d_{B} z^{2}+2 d_{B}^{2} z+2 d_{B}^{3}\right)\right]_{d_{i}}^{d_{j}} \\
& \equiv I\left(d_{B} ; d_{i}, d_{j}\right) .
\end{aligned}
$$

Hence, the final expression for the total number of dwarfs in a truncated cone with the minor and major bases at heliocentric distances $d_{1}$ and $d_{2}$, respectively, is:

$N_{i}\left(r_{1}, r_{2}\right)=\mathcal{S}\left[n_{A_{+, i}} I\left(d_{B_{+}} ; d_{1}, d_{*}\right)+n_{A_{-, i}} I\left(d_{B_{-}} ; d_{*}, d_{2}\right)\right]$.

\subsection{Possible late-type contaminants}

We have studied the contribution to contamination in photometric searches for very red sources by field dwarfs with spectral types from M3V to T8V. This election is based, on the one hand, on the earliest spectral type of a brown dwarf in a 1 Ma-old star-forming region, which is about M5, and the large-amplitude photometric variability that can be present in the brightest young substellar objects, which can be as large as 0.7 mag (Caballero et al. 2006). The maximum colour variation observed could be even larger than the amplitude of photometric variability. Because of that, if the surveys in each passband are not simultaneous, very young variable brown dwarfs can display colours of objects a few sub-types earlier than its actual spectral type. On the other hand, the late-type bound is set by the latest-type brown dwarfs observed to date (e.g. Burgasser et al. 2000; Tinney et al. 2005; Looper et al. 2007; Lodieu et al. 2007a; 
Table 3. Characteristics of possible late-type field dwarf contaminants from the literature ${ }^{a}$.

\begin{tabular}{lccccc}
\hline \hline $\begin{array}{l}\text { Spectral } \\
\text { type }\end{array}$ & $\begin{array}{c}M_{I} \\
(\mathrm{mag})\end{array}$ & $\begin{array}{c}I-J \\
(\mathrm{mag})\end{array}$ & $\begin{array}{c}J-K_{\mathrm{s}} \\
(\mathrm{mag})\end{array}$ & $\begin{array}{c}n_{\odot} \\
\left(10^{-3} \mathrm{pc}^{-3}\right)\end{array}$ & $\begin{array}{c}d_{I=25}^{b} \\
(\mathrm{pc})\end{array}$ \\
\hline M3-4 V & 9.07 & 1.83 & 0.84 & 19.6 & 7700 \\
M4-5 V & 10.14 & 1.80 & 0.86 & 16.3 & 5700 \\
M5-6 V & 11.91 & 2.47 & 0.90 & 9.81 & 3100 \\
M6-7 V & 12.90 & 2.72 & 0.93 & 4.90 & 2200 \\
M7-8 V & 14.16 & 3.24 & 0.98 & 1.907 & 1300 \\
M8-9 V & 14.68 & 3.54 & 1.06 & 1.285 & 1100 \\
M9-L0 V & 15.23 & 3.80 & 1.12 & 0.768 & 840 \\
L0-1 V & 15.27 & 3.55 & 1.27 & 0.637 & 820 \\
L1-2 V & 15.69 & 3.50 & 1.38 & 0.861 & 680 \\
L2-3 V & 16.19 & 3.62 & 1.43 & 1.000 & 550 \\
L3-4 V & 16.67 & 3.72 & 1.49 & 0.834 & 450 \\
L4-5 V & 17.14 & 3.81 & 1.54 & 0.819 & 360 \\
L5-6V & 17.61 & 3.90 & 1.60 & 0.869 & 290 \\
L6-7 V & 18.07 & 4.06 & 1.66 & 0.785 & 240 \\
L7-8 V & 18.52 & 4.12 & 1.71 & 0.644 & 190 \\
L8-9 V & 18.90 & 4.22 & 1.81 & 0.462 & 160 \\
L9-T0 V & 18.95 & 4.40 & 1.73 & 0.308 & 160 \\
T0-1 V & 19.01 & 4.63 & 1.58 & 0.220 & 160 \\
T1-2 V & 19.04 & 4.94 & 1.17 & 0.241 & 150 \\
T2-3 V & 19.04 & 5.19 & 0.81 & 0.406 & 150 \\
T3-4 V & 19.07 & 5.23 & 0.51 & 0.788 & 150 \\
T4-5 V & 19.22 & 5.01 & 0.29 & 1.42 & 140 \\
T5-6 V & 19.61 & 4.76 & 0.11 & 2.38 & 120 \\
T6-7 V & 20.35 & 5.25 & 0.00 & 3.45 & 85 \\
T7-8 V & 21.60 & 5.50 & -0.06 & 4.82 & 48 \\
\hline
\end{tabular}

${ }^{a}$ The sources of the $M_{I}$ absolute magnitude, $I-J$, and $J-K_{\mathrm{s}}$ colours, and spatial densities are obtained or derived by us from data in the literature (Kirkpatrick et al. 1994; Dahn et al. 2002; Hawley et al. 2002; Cruz et al. 2003; Tinney et al. 2003; Vrba et al. 2004; Bochanski et al. 2007; Burgasser 2007; West et al. 2008). See the text for further details. ${ }^{b} d_{I=25}$ is the heliocentric distance of a dwarf with an apparent magnitude $I=25.0 \mathrm{mag}$, assuming an interstellar extinction proportional to the heliocentric distance. At the end of Sect. 2.4 there is a brief discussion on this assumption.

Warren et al. 2007). The total number of contaminants in the survey is the sum of the number of field dwarfs of spectral type $i$ in the truncated cones of search:

$N=\sum_{i=1}^{\text {Num. }} N_{i}\left(r_{1, i}, r_{2, i}\right)$,

( $i=\mathrm{M} 3-4 \mathrm{~V}, \mathrm{M} 4-5 \mathrm{~V}, \mathrm{M} 5-6 \mathrm{~V} \ldots \mathrm{T} 7-8 \mathrm{~V})$.

The Johnson $I$-band absolute magnitudes, $I-J$, and $J-K_{\mathrm{s}}$ colours ( $J$ and $K_{\mathrm{s}}$ in the 2MASS system), and local spatial densities from the literature of the 25 considered intervals of spectral types are given in Table 3. For the M3-L1 dwarfs, we used the tabulated values from West et al. (2008) of $i_{\text {SDSS }}-J$, then calculated the $I-i_{\text {SDSS }}$ corrections using the template spectra of Bochanski et al. (2007) to derive the $I-J$ colours. Absolute $I$-band magnitudes were computed from absolute $J$-band magnitudes compiled by Hawley et al. (2002) and using our $I-J$ values. $J-K_{\mathrm{s}}$ colours are also from West et al. (2008). For the L8-T0 dwarfs, $J-K_{\mathrm{s}}$ values are from DwarfArchives.org, taking the average for objects with uncertainties $\delta\left(J-K_{\mathrm{S}}\right)<$ $0.1 \mathrm{mag}$ and not known to be binary. The absolute $I$ and $I-J$ colours are based on the polynomial relations of Tinney et al. ( 2003 - note the backtrack in $I-J$ colours). The remaining $I J K_{\mathrm{s}}$ photometry was taken from Dahn et al. (2002) and Vrba et al. (2004).

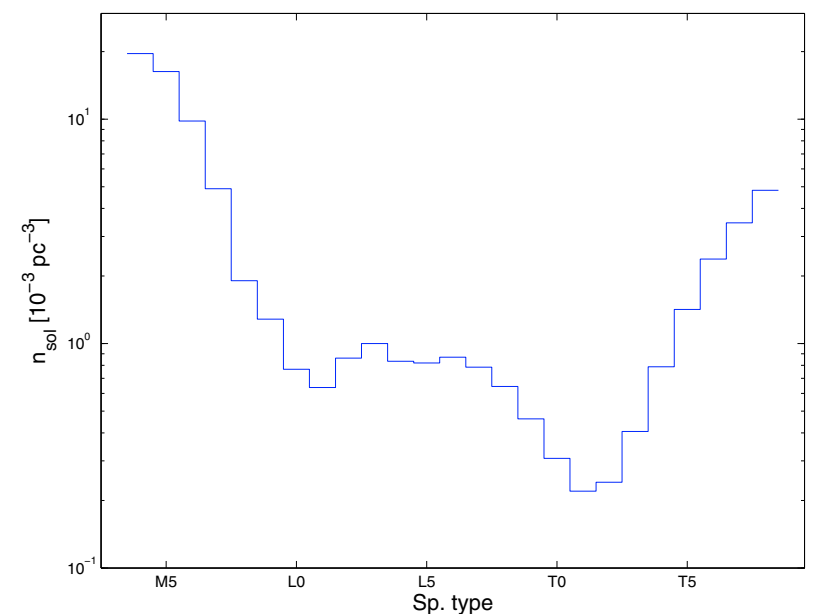

Fig. 3. Local spatial density of ultracool dwarfs as a function of spectral type from the data in Table 3.

The local spatial densities $\left(n_{\odot}\right)$ and the spatial densities at $R=R_{\odot}$ and $Z=0\left(n_{0}\right)$ are related by Eq. (8). A pictorical representation of $n_{\odot}$ versus spectral type is given in Fig. 3 . Spatial densities of M3-7 and M7-L0 dwarfs are the values measured by Kirkpatrick et al. (1994) and Cruz et al. (2003), respectively. For L0-T8 dwarfs, we used predicted values from Burgasser (2007) based on a Monte Carlo simulation assuming a mass function $\mathrm{d} N / \mathrm{d} M \propto M^{-0.5}$ (similar to estimates from various star-forming regions - e.g. Luhman et al. 2000; Caballero et al. 2007), a normalisation of $0.0037 \mathrm{pc}^{-3}$ over the range $0.09-0.10 M_{\odot}$ (Reid et al. 1997), a binary fraction of $10 \%$, and a binary mass ratio distribution of $P(q) \propto q^{4}$ (where $q \equiv M_{2} / M_{1}$ ). These values are consistent with empirical space density estimates by Burgasser et al. (2002), Cruz et al. (2007), and Metchev et al. (2008), and predicted densities by Deacon \& Hambly (2006), although they differ significantly from predictions by Nakajima (2005) for L5-T7 dwarfs.

Using the expression $I-M_{I}=5 \log d-5+A_{I}$, a deep survey with a limiting magnitude $I_{\mathrm{lim}}=25.0 \mathrm{mag}$ would be able to detect a T5-6 dwarf at a heliocentric distance $d \sim 120 \mathrm{pc}$ and an M 5-6 dwarf at about $3.1 \mathrm{kpc}$ from the Sun (see the last column in Table 3). In the direction of the Pleiades, such a hypothetical M-type "field" dwarf would be located at about $1.9 \mathrm{kpc}$ below the Galactic plane at almost $z=6 \times h_{z}$. In this location, the spatial density of thin-disc objects of any spectral type is about $10^{-3}$ times the local density $n_{\odot}$. From this example, the necessity of the integration along the cone of search using a non-uniform density is clearly evident. Following this assumption, we should also use an interstellar extinction that does not increase linearly with heliocentric distance. Instead, it should be proportional to the actual interstellar dust content. In our model, we assume that the Galactic dust content has much wider length and height scales than for stars.

\section{A practical application: $L$ and $T$ dwarf contamination in $\sigma$ Orionis}

\subsection{Preliminaries}

The $\sigma$ Orionis cluster $(\sim 3 \mathrm{Ma}, \sim 385 \mathrm{pc})$ contains one of the most well known substellar populations, from the hydrogen burning mass limit down to a few Jupiter masses (see a bibliographic review in Caballero 2008b). It could be the most favourable site known to date for studying the opacity mass limit for 
formation of objects via fragmentation in molecular clouds, that may lie just below $0.003 M_{\odot}$ (Rees 1976; Bate et al. 2003, and references therein). Even accounting for the L-type cluster members and candidates in the Pleiades (Martín et al. 1998; Dobbie et al. 2002b; Moraux et al. 2003; Bihain et al. 2006), Chamaeleon and Lupus (Allers et al. 2006; Jayawardhana \& Ivanov 2006), $\sigma$ Orionis contains by far the largest number of L-type objects with spectroscopy (12 - Zapatero Osorio et al. 1999, 2000; Martín et al. 2001; Barrado y Navascués et al. 2001, 2003). While there is reasonable criticism about the cluster membership of some of the faintest objects (e.g. S Ori 47, L1.5 $\pm 0.5-$ McGovern et al. 2004), several of them are known to be extremely young based on near-infrared excess and extraordinary $\mathrm{H} \alpha$ emission of up to $-700 \AA$ (S Ori 55 , S Ori $71-$ Barrado y Navascués et al. 2002; Zapatero Osorio et al. 2002a; Caballero et al. 2007) or flux excess in the IRAC Spitzer bands (Zapatero Osorio et al. 2007b; Scholz \& Jayawardhana 2007). $\sigma$ Orionis also possesses the only extremely young T-type cluster member candidate identified so far (S Ori 70; it was introduced in Sect. 1 - Casewell et al. 2007 and Bouvier et al. 2008, identified $\mathrm{T}$ dwarf candidates and cluster members in the Pleiades [ 120 Ma] and the Hyades [ 600 Ma]).

The contamination by field late-M dwarfs in the cluster is well constrained. Caballero et al. (2007) identified 30 objects as bona fide $\sigma$ Orionis members with features of extreme youth amongst 49 candidate cluster members fainter than $I=16$ mag selected from an $I$ versus $I-J$ diagram. They also reported the detection of two L-type field dwarf candidates in the direction of the cluster, even fainter (and much more distant) than Cuby et al. (1999)'s T dwarf. The ratio between very low-mass confirmed and candidate cluster members in $\sigma$ Orionis is comparable to that in the Orion Nebula Cluster (Lucas et al. 2001; Slesnick et al. 2004), but much higher than in other older open clusters such as the Praesepe (Pinfield et al. 1997, 2003; Adams et al. 2002; González-García et al. 2006) or the Hyades (Reid \& Hawley 1999; Gizis et al. 1999; Dobbie et al. 2002a). In any case, some of the faintest substellar member candidates in $\sigma$ Orionis have no astrometric confirmation or irrefutable youth signatures. Therefore, while there are no new data on cluster membership, it is necessary to accurately determine the number of possible fore- and background contaminants among selected photometric cluster member candidates for investigating the cluster Initial Mass Function. We applied our Galaxy and late-type dwarf data and mathematical tools to estimate the number of fore- and background $\mathrm{L}$ and $\mathrm{T}$ dwarfs as a function of depth in a hypothetical survey area of $1 \mathrm{deg}^{2}\left(\mathcal{S} \approx 0.00030 \mathrm{rad}^{2}\right)$ in the $\sigma$ Orionis cluster.

We assumed that the cluster member selection was based exclusively on $I-X$ colours (Johnson $I$ ), where $X$ represents a near-infrared passband (e.g. $J H K_{\mathrm{s}}$ ). The $X$-band observations were sufficiently deep to detect within the completeness levels all the L- or T-type object identified in the $I$-band images ${ }^{1}$. The combination of a red optical filter and a near-infrared one is an effective way (i.e. in telescope time and simplicity) of selecting very faint red objects in young open clusters. Although an $I-K_{\mathrm{s}}$-based search would identify late-L-type objects more accurately, the increase in thermal background at $2 \mu \mathrm{m}$ of nearinfrared detectors favours the use of the $J$ passband, especially for T-type objects (that have quite blue $J-K_{\mathrm{s}}$ colours); a

${ }^{1}$ For example, the completeness magnitudes in the representative survey of Caballero et al. (2007) were $I_{\text {compl. }}=23.3 \mathrm{mag}, J_{\text {compl. }}=$ $20.6 \mathrm{mag}$, allowing the detection of all the objects within the completeness levels in the optical redder than $I-J=2.7 \mathrm{mag}(\sim \mathrm{M} 7 \mathrm{~V})$.
Table 4. Number of expected field L- and T-type dwarfs in a $1 \mathrm{deg}^{2}$-area survey towards $\sigma$ Orionis as a function of the Johnson $I$-band magnitude and spectral type interval ${ }^{a}$.

\begin{tabular}{lcccc}
\hline \hline $\begin{array}{l}\Delta I \\
(\mathrm{mag})\end{array}$ & L0-5V & L5-T0V & T0-5V & T5-8V \\
\hline $21.0-22.0$ & $1.1_{-0.4}^{+0.5}$ & $0.049_{-0.019}^{+0.034}$ & $0.012_{-0.005}^{+0.009}$ & $0.008_{-0.003}^{+0.007}$ \\
$22.0-23.0$ & $3.9_{-1.4}^{+1.8}$ & $0.19_{-0.08}^{+0.14}$ & $0.05_{-0.02}^{+0.03}$ & $0.030_{-0.014}^{+0.027}$ \\
$23.0-24.0$ & $12_{-4}^{+5}$ & $0.7_{-0.3}^{+0.5}$ & $0.18_{-0.08}^{+0.14}$ & $0.12_{-0.06}^{+0.11}$ \\
$24.0-25.0$ & $36_{-13}^{+14}$ & $2.5_{-1.0}^{+1.7}$ & $0.7_{-0.3}^{+0.5}$ & $0.5_{-0.2}^{+0.4}$ \\
$25.0-26.0$ & $90_{-30}^{+30}$ & $8_{-3}^{+4}$ & $2.5_{-1.1}^{+1.8}$ & $1.8_{-0.8}^{+1.5}$ \\
$26.0-27.0$ & $180_{-80}^{+60}$ & $24_{-10}^{+14}$ & $8_{-3}^{+5}$ & $6_{-3}^{+6}$ \\
$27.0-28.0$ & $270_{-130}^{+100}$ & $60_{-30}^{+30}$ & $25_{-11}^{+16}$ & $20_{-10}^{+17}$ \\
$28.0-29.0$ & $300_{-170}^{+140}$ & $130_{-60}^{+60}$ & $60_{-30}^{+40}$ & $60_{-30}^{+50}$ \\
\hline
\end{tabular}

${ }^{a}$ Poissonian localized overdensities are not taken into account.

compromise can be reached by developing an $I-H$ survey. Likewise, imaging at passbands bluewards of $I$ is prohibitive due to the extreme dimness of ultracool cluster member candidates at these wavelengths (e.g. a cluster member with $I=24 \mathrm{mag}$ is expected to have $R \approx 27-28 \mathrm{mag}$ ). Finally, the number of interlopers with pure optical $(R-I, I-Z)$ and pure infrared $\left(J-K_{\mathrm{s}}\right)$ passbands is larger than with a combination of red optical and near-infrared filters, because of the larger slope in the spectral energy distribution of young late-type objects at the optical/infrared boundary (i.e. redder $I-J, I-K_{\mathrm{s}}$ colours).

As explained in Sect. 2.3.1, one step in the integration of the number of contaminants is deciding the minimum and maximum heliocentric distances $d_{1}$ and $d_{2}$ specified in Eq. (14). In computing the heliocentric distance of an object of apparent and absolute magnitudes $I$ and $M_{I}$, we have to find the root of the smooth function $f(d)=5 \log d-5+A_{I}-\left(I-M_{I}\right)$, where the extinction $A_{I}=0.482 A_{V}$ is proportional to the distance $\left(A_{I}=a d\right)$ and $A_{V}=3.09 E(B-V)$ (Rieke \& Lebofski 1985). The constant $a$ depends on the colour excess $E(B-V)_{\star}$ and distance $d_{\star}$ towards $\sigma$ Orionis (e.g. Lee 1968; Caballero 2008a). Quantitatively: $E(B-V)=E(B-V)_{\star}\left(d / d_{\star}\right)$. To find the root, we used the Newton method $\left(d_{n+1}=d_{n}-f(n) / f^{\prime}(n), n=0,1\right.$, $2 \ldots)$, which converges rapidly in 3-6 iterations with a suitable initial value of $d_{0}$.

In Table 4, we summarize our results and provide the number of L0-T8V-type dwarfs in each of the 1.0 mag-width "strips". As discussed below, not all the late-type field dwarfs in the survey area are true contaminants (e.g. L-type dwarfs do not contaminate the $\sigma$ Orionis $I$ versus $I-J$ colour-magnitude diagram at $I \gtrsim 24.5 \mathrm{mag}$ ). The uncertainties originate in the errors in the parameters of the thin disc (Table 1), the cluster heliocentric distance $(d=330-440 \mathrm{pc})$, the spectral type-absolute magnitude relation, the colour excess, and the local spatial densities. We use conservative errors of $0.2 \mathrm{mag}$ and $0.02 \mathrm{mag}$ for the spectral type- $M_{I}$ relation and the interstellar extinction, respectively. Variations in the solar galactocentric distance $R_{\odot}$ barely affect the results. The most important contributions to the error are the radial scale length $h_{\mathrm{r}}$, which is the Galactic parameter with the largest intrinsic uncertainty $(>40 \%)$, and the local spatial densities. The mass function index that Burgasser (2007) used for deriving $n_{\odot}\left(\alpha=+0.5 ; \mathrm{d} N / \mathrm{d} M \propto M^{-\alpha}\right)$ may be too large according to observational results (Lodieu et al. 2007b; Metchev et al. 2008). A flatter substellar mass function $(\alpha \approx 0.0)$ would lead to lower local spatial densities and, therefore, lower contamination rates. The choice of the single power-law mass 
function of Burgasser (2007) (from where L0-T8V local densities in Table 1 were obtained) appears more reasonable, since it explains the increase in the mass function derived by many other authors for the planetary regime (see Sect. 1). The measurements of Metchev et al. (2008) are consistent with those of Burgasser (2007) within the $95 \%$ confidence limits. Maximum differences with other determinations of substellar densities in the literature are approximately $20 \%$ with respect to our measurements. Our estimation of the uncertainties in $n_{\odot}$ is, however, more conservative: the maximum and minimum surface densities are determined by using very different indices in the local mass function, $\alpha=0$ and +1 (see Table 5 in Burgasser 2007). The error in $n_{\odot}$ depends strongly on spectral type, and ranges from $\sim 12 \%$ at $\mathrm{L} 0-1 \mathrm{~V}$ to $\sim 50 \%$ at $\mathrm{T} 7-8 \mathrm{~V}$.

Our computations surpass and complement the estimate of contamination in $\sigma$ Orionis by Caballero et al. (2007). The number of interlopers shown in Table 4 are consistent with those given in Caballero et al. (2007), accounting for the different survey areas, input absolute magnitudes $M_{I}$, and local densities $n_{\odot}$ (especially in the L5-T7V interval), and width of strips (i.e. $I-J$ colour). They estimated that $\sim 4$ L-type dwarf contaminants ( $\sim 2$ early, $\sim 2$ late) populated their least-massive bin of the Initial Mass Function, which contained 11 planetary-mass object candidates (i.e. the contamination rate was 36\%); our results support those calculations. A coarse extrapolation towards fainter magnitudes of surface densities provided by Burgasser (2007) also matches our results, although he did not account for the Galactic structure. Values similar to those in Table 4 are expected for $1 \mathrm{deg}^{2}$-wide surveys of any kind (young open clusters, extragalactic) at intermediate galactic and high latitudes of the same magnitude depth.

\subsection{Discussion}

In summary, the number of ultracool dwarfs in deep surveys from Table 4 is larger for earlier spectral types and the faintest magnitude intervals. The contamination by $\mathrm{T}$ dwarfs is low for survey depths $I \lesssim 24 \mathrm{mag}$. Surveys deeper than this value are affected strongly by L- and T-type dwarf interlopers. From Fig. 4, the behaviour of the $\sigma$ Orionis luminosity function is unclear at $I \gtrsim 24 \mathrm{mag}$. The deepest available optical surveys of young clusters, in general, and of $\sigma$ Orionis, in particular, reach limiting magnitudes of $I=24-25 \mathrm{mag}$ (Caballero et al. 2007, and references therein - see also Comerón \& Claes 2004, for a very deep near-infrared survey in Chamaeleon I), although some ultra-deep extragalactic surveys reach 2-4 mag fainter. The magnitudes $(I \approx 22.8$ and $23.2 \mathrm{mag})$ and measured spectral types (L5.0 \pm 1.0 and L3.5 \pm 2.0 - Barrado y Navascués et al. 2001; Martín et a al. 2001) of the faintest cluster objects with unambiguous youth features, S Ori 65 and S Ori 60 (they display flux excess at 8.0 and/or $5.8 \mu \mathrm{m}$ - Zapatero Osorio et al. 2007b; Scholz \& Jayawardhana 2008) suggest that the $\mathrm{L}-\mathrm{T}$ transition in the cluster occurs at about $I \sim 23.5$ mag (field T0-1V dwarfs are about $1.5 \mathrm{mag}$ fainter than L5-6V ones however, this assumption might not be correct necessarily for low-gravity sources due to pressure effects on alkali line absorption). S Ori 69 , the second faintest cluster member candidate $(I \approx 23.9 \mathrm{mag}$ ) has a tentative "T0:" spectral type determined from a near-infrared spectrum (Martín et al. 2001), which supports our estimate (the semi-colon indicates uncertainty in the classification). Therefore, fainter cluster members are expected to have $\mathrm{T}$ spectral types, colours $I-J \gtrsim 4.5 \mathrm{mag}$, and most probable masses derived from state-of-the-art theoretical

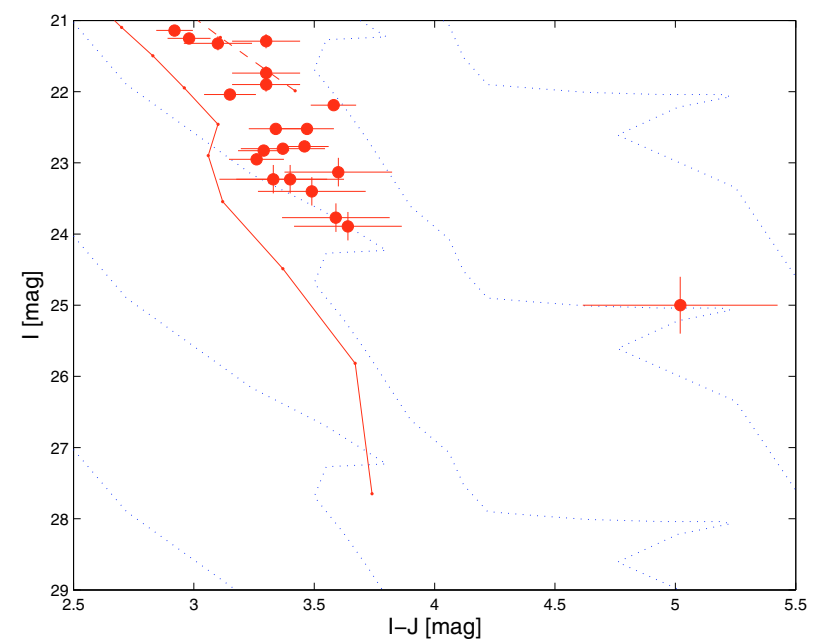

Fig. 4. $I$ vs. $I-J$ colour-magnitude diagram of the $\sigma$ Orionis cluster. Large filled circles with error bars: cluster members and candidates (the faint red outlier is the T-type object S Ori 70); solid and dashed lines: COND03 and DUSTY00 3 Ma-old isochrones, respectively, shifted to $d=385$ pc (Chabrier et al. 2000; Baraffe et al. 2003); dotted lines: dwarf sequence in Table 3 shifted by distance moduli $m-M=3.0$, $6.0,9.0,12.0$, and $15.0 \mathrm{mag}$, from top to bottom. The faintest data point of the COND03 theoretical isochrone $(I-J \approx 3.0 \mathrm{mag}, I \approx 29.0 \mathrm{mag}$ ) corresponds to an $1.0 \mathrm{M}_{\mathrm{Jup}}$-mass object. L and T field dwarfs have approximate colours redder than $I-J \approx 3.5$ and 4.5 mag.

models of $M \lesssim 0.006 M_{\odot}$ (we do not discuss in this paper the cluster membership status of S Ori 70).

The actual number of $\sigma$ Orionis cluster members and candidates of L0-5 and L5-T0 spectral types at the respective magnitudes $I \sim 21.0-22.5$ and $22.5-24.0 \mathrm{mag}$ in a survey is higher than the expected number of dwarf contaminants of the same spectral type (e.g. Zapatero Osorio et al. 2000). Although significant, the difference requires confirmation by a careful spectroscopic/photometric/astrometric follow-up because fluctuations about the mean could mask the data. A change in space density of contaminants towards $\sigma$ Orionis by some Poissonian factor would easily cause the L-type contamination rate to increase from 36 to $\sim 70 \%$ or higher. Localized overdensities of dwarfs in the Galactic thin disc are not unusual (e.g. Jurić et al. 2008, and references therein). Field $\mathrm{L}$ dwarfs fainter than $I \approx 24.5 \mathrm{mag}$ stop contaminating the $\sigma$ Orionis colour-magnitude diagram at $I-J \gtrsim 4.5 \mathrm{mag}$. Since the interstellar reddening is appreciable only at large heliocentric distances $\left(A_{I}=a d\right.$, being $\left.a \approx 2 \times 10^{-4} \mathrm{mag} \mathrm{pc}^{-1}\right)$, where the spatial density is extremely low, contamination by M dwarfs is also unexpected.

Surveys with completeness magnitudes $I_{\text {compl. }} \approx 27-28 \mathrm{mag}$ should be required to search for the opacity mass limit (at $M \lesssim 0.003 M_{\odot}$ ), and field $\mathrm{T}$ dwarfs are expected to be the major colour-selected contaminants at these magnitudes. While the contamination by $\mathrm{T}$ dwarfs in the brightest magnitude intervals in Table 4 is low with respect to the contamination by L dwarfs (by a factor $\sim 100$ ), it dramatically increases at the faintest magnitude intervals. For example, for $\Delta I=28.0-29.0 \mathrm{mag}$, the number of L0-L5V dwarf contaminants reaches the maximum limit of $\sim 300$ objects (i.e. the survey is so deep that it is able to detect almost all the early L dwarfs of the Galaxy in that direction), whereas there are only 3.6 times less $\mathrm{T}$ dwarfs than $\mathrm{L}$ dwarfs for the same $\Delta I$. From the data in Table 4, we expect about $\sim 45$ T-type dwarfs in the foreground of $\sigma$ Orionis in an ultra-deep $(I \approx 27-28 \mathrm{mag})$ survey. However, the most optimistic estimations of a large index $\alpha=+0.6$ of the cluster 
mass spectrum (Caballero et al. 2007) extrapolated towards only $0.001 M_{\odot}$ predict $\$ 30$ planetary-mass objects with $\mathrm{T}$ spectral types in $\sigma$ Orionis (Zapatero Osorio, priv. comm.). By assuming less favourable conditions, we forecast no more than $\sim 10$ T-type cluster members. These values depend on assumptions that require confirmation, e.g. the distance and age of the cluster, the actual value of the slope of the mass spectrum at Jovian masses, or the validity of theoretical evolutionary tracks of lowmass stars and brown dwarfs at very young ages and low masses (Baraffe et al. 2002). For example, Fig. 4 shows that COND03 models (Baraffe et al. 2003), which are especially useful for the T-type domain in the field, predict blue $I-J$ colours for the least massive hypothetical $\sigma$ Orionis members (with masses down to $\sim 1.0 M_{\text {Jup }}$ ). Accurate theoretical modelling of low gravity, low temperature spectra is work still in progress.

Even with the largest current ground facilities $(2 \times 10.0 \mathrm{~m}$ Keck Observatory, $10.4 \mathrm{~m}$ Gran Telescopio Canarias), the necessary follow-up of an ultra-deep survey for studying the opacity mass limit in $\sigma$ Orionis would require a tremendous, prohibitive, observational effort. For example, the low-resolution near-infrared spectrum of the $\sim$ T6 object S Ori $70(I=25.0 \pm$ $0.4 \mathrm{mag}, J=19.98 \pm 0.06 \mathrm{mag}$ ) was acquired for a total exposure time of $4800 \mathrm{~s}$ with NIRSPEC at the Keck II (Martín \& Zapatero Osorio 2003), although it was of low signal-to-noise ratio. Sources with $I \approx 27-28$ mag would need correspondingly longer exposure times, of up to an entire night. Therefore, spectroscopy of roughly half of the selected cluster member candidates in such an ultra-deep survey ( $~ 220$ targets) would require about one month of observing time at the Keck II. Shallower surveys ( $I \lesssim 26 \mathrm{mag}$ ) and their corresponding follow-ups are, however, much less affected by T-dwarf contamination ( $\sim 5$ interlopers in a $1 \mathrm{deg}^{2}$-wide survey) and would require a far more reasonable amount of time to be accomplished. The photometric selection becomes more stringent and may lower the contamination level when several colour-magnitude diagrams, colourcolour diagrams, and $\mathrm{CH}_{4}$-on and off photometry are combined. A survey with at least three broad filters (e.g. $I J K_{\mathrm{S}}$ or $\left.z^{\prime} H[4.5]\right)^{2}$ and the two narrow methane filters, although multiplying the total exposure time by a factor $\sim 5-6$ with respect to an $I J$ survey, may represent an intermediate solution.

\section{Summary}

Late-type field dwarfs are the most important contributors to contamination among faint, young, cluster member candidates selected from colour-magnitude diagrams. The number of such interlopers in deep photometric surveys in clusters is of significant importance to the study of the substellar Initial Mass Function. We provide a pseudo-algorithm, an expression for the integrated number of field dwarfs at certain heliocentric distance and galactic coordinates (assuming an accurate linear approximation in the structure of the Galactic thin disc), and the absolute magnitudes, colours, and local spatial densities of M3-T8 dwarfs to compute the number of field late-type contaminants in deep surveys excluding the Galactic plane.

We have applied our tools and data to a hypothetical ultradeep $\left(I_{\text {compl. }}=29 \mathrm{mag}\right)$ survey towards the young $(\sim 3 \mathrm{Ma})$ $\sigma$ Orionis cluster. We predict a rather low contamination rate of $\mathrm{L}$ and $\mathrm{T}$ field dwarfs with an appropriately defined selection criterion up to $I=25-26 \mathrm{mag}$. The number of contaminants at fainter magnitudes, where the opacity mass limit is expected to

${ }^{2}$ [4.5] is the $4.5 \mu \mathrm{m}$ IRAC passband at the Spitzer Space Observatory, still useful during post-cryo phase. lie, is however very high and may preclude photometric, spectroscopic, and astrometric follow-up in reasonable amounts of time with current facilities.

An increase in the contamination by T-type dwarfs in ultraand very deep surveys is not only important for $\sigma$ Orionis, in particular, and star-forming regions, in general, but also for extragalactic surveys of $z \gtrsim 6$ quasars, whose colours resemble those of $\mathrm{T}$ dwarfs.

Acknowledgements. We thank the anonymous referee for his/her helpful report, I. Baraffe for providing us 3 Ma-old Lyon tracks, C. Halliday for revising the manuscript, and M. Cornide and M. R. Zapatero Osorio for helpful comments. J.A.C. formerly was an Alexander von Humboldt Fellow at the MPIA and currently is an Investigador Juan de la Cierva at the UCM. R.K. is a granted Ph.D. student at the MPIA. Partial financial support was provided by the Universidad Complutense de Madrid and the Spanish Ministerio Educación y Ciencia under grant AyA2005-02750 of the Programa Nacional de Astronomía y Astrofísica and by the Comunidad Autónoma de Madrid under PRICIT project S-0505/ESP-0237 (AstroCAM). Research has benefitted from the M, L, and T dwarf compendium housed at http://DwarfArchives.org and maintained by Chris Gelino, Davy Kirkpatrick, and Adam Burgasser.

\section{References}

Adams, J. D., Stauffer, J. R., Skrutskie, M. F., et al. 2002, AJ, 124, 1570

Allers, K. N., Kessler-Silacci, J. E., Cieza, L. A., \& Jaffe, D. T. 2006, ApJ, 644, 364

Allers, K. N., Jaffe, D. T., Luhman, K. L., et al. 2007, ApJ, 657, 511

Ardila, D., Martín, E. L., \& Basri, G. 2000, AJ, 120, 479

Bahcall, J. N., \& Soneira, R. M. 1980, ApJS, 44, 73

Bahcall, J. N., \& Soneira, R. M. 1984, ApJS, 55, 67

Baraffe, I., Chabrier, G., Allard, F., \& Hauschildt, P. H. 2002, A\&A, 382, 563

Baraffe, I., Chabrier, G., Barman, T., Allard, F., \& Hauschildt, P. H. 2003, A\&A, 402, 701

Barrado y Navascués, D., Zapatero Osorio, M. R., Béjar, V. J. S., et al. 2001, A\&A, 377, L9

Barrado y Navascués, D., Zapatero Osorio, M. R., Martín, E. L., et al. 2002, A\&A, 393, L85

Barrado y Navascués, D., Béjar, V. J. S., Mundt, R., et al. 2003, A\&A, 404, 171 Bate, M. R., Bonnell, I. A., \& Bromm, V. 2003, MNRAS, 339, 577

Béjar, V. J. S., Martín, E. L., Zapatero Osorio, M. R., et al. 2001, ApJ, 556, 830 Bihain, G., Rebolo, R., Béjar, V. J. S., et al. 2007, ApJ, 657, 511 2006, A\&A, 458,805

Blaauw, A., Gum, C. S., Pawsey, J. L., \& Westerhout, G. 1960, MNRAS, 121, 123

Bochanski, J. J., West, A. A., Hawley, S. L., \& Covey, K. R. 2007, AJ, 133, 531

Bouvier, J., Stauffer, J. R., Martín, E. L., et al. 1998, A\&A, 336, 490

Bouvier, J., Kendall, T., Meeus, G., et al. 2008, A\&A, 481, 661

Burgasser, A. 2007, ApJ, 659, 655

Burgasser, A., Kirkpatrick, J. D., Cutri, R. M., et al. 2000, ApJ, 531, L57

Burgasser, A., Kirkpatrick, J. D., Brown, M. E., et al. 2002, ApJ, 564, 421

Burgasser, A. J., Kirkpatrick, J. D., McGovern, M. R., et al. 2004, ApJ, 604, 827

Burrows, A., Marley, M., Hubbard, W. B., et al. 1997, ApJ, 491, 856

Caballero, J. A. 2007, ApJ, 667, 520

Caballero, J. A. 2008a, MNRAS, 383, 750

Caballero, J. A. 2008b, A\&A, 478, 667

Caballero, J. A., Béjar, V. J. S., Rebolo, R., \& Zapatero Osorio, M. R. 2004, A\&A, 424, 857

Caballero, J. A., Martín, E. L., Zapatero Osorio, M. R., et al. 2006, A\&A, 445, 143

Caballero, J. A., Béjar, V. J. S., Rebolo, R., et al. 2007, A\&A, 470, 903

Casewell, S. L., Dobbie, P. D., Hodgkin, S. T., et al. 2007, MNRAS, 378, 1131

Chabrier, G., Baraffe, I., Allard, F., \& Hauschildt, P. 2000, ApJ, 542, 464

Chen, B., Stoughton, C., Smith, J. A., et al. 2001, ApJ, 553, 184

Comerón, F., \& Claes, P. 2004, ApJ, 602, 298

Cruz, K. L., Reid, I. N., Liebert, J., Kirkpatrick, J. D., \& Lowrance, P. J. 2003, AJ, 126, 2421

Cruz, K. L., Reid, I. N., Kirkpatrick, J. D., et al. 2007, AJ, 133, 439

Cuby, J. G., Saracco, P., Moorwood, A. F. M., et al. 1999, A\&A, 349, L41

Dahn, C. C., Harris, H. C., Vrba, F. J., et al. 2002, AJ, 124, 1170

Deacon, N. R., \& Hambly, N. C. 2006, MNRAS, 371, 1722

Dobbie, P. D., Kenyon, F., Jameson, R. F., et al. 2002a, MNRAS, 329, 543

Dobbie, P. D., Kenyon, F., Jameson, R. F.et al. 2002b, MNRAS, 335, 687

Eisenhauer, F., Schödel, R., Genzel, R., et al. 2003, ApJ, 597, L121

Faber, S. M., Burstein, D., Tinsley, B., \& King, I. R. 1976, AJ, 81, 45 
Feltzing, S., Bensby, T, \& Lundström, I. 2003, A\&A, 397, L1

Foster, J. B., Román-Zúñiga, C. G., Goodman, A. A., Lada, E. A., \& Alves, J. 2008, ApJ, 674, 831

Fuhrmann, K. 1998, A\&A, 338, 161

Gilmore, G. 1984, MNRAS, 207, 223

Gilmore, G., \& Reid, N. 1983, MNRAS, 202, 1025

Gilmore, G., King, I., \& van der Kruit, P. 1990, The Milky Way as a Galaxy (University Science Books)

Gizis, J. E., Reid, I. N., \& Monet, D. G. 1999, AJ, 118, 997

Gizis, J. E., Monet, D. G., Reid, I. N., et al. 2000, AJ, 120, 1085

González-García, B. M., Zapatero Osorio, M. R., Béjar, V. J. S., et al. 2006, A\&A, 460, 799

Hambly, N. C., Hodgkin, S. T., Cossburn, M. R., \& Jameson, R. F. 1999, MNRAS, 303, 835

Hambly, N. C., MacGillivray, H. T., Read, M. A., et al. 2001, MNRAS, 326, 1279

Hawley, S. L., Covey, K. R., Knapp, G. R., et al. 2002, AJ, 123, 3409

Jayawardhana, R., \& Ivanov, V. D. 2006, Science, 313, 1279

Jeffries, R. D., Naylor, T., Devey, C. R., \& Totten, E. J. 2004, MNRAS, 351, 140

Jurić, M., Ivezić, Z., Brooks, A., et al. 2008, ApJ, 673, 864

Karaali, S. 2006, AN, 327, 97

Kendall, T. R., Tamura, M., Tinney, C. G., et al. 2007, 466, 1059

Kerr, F. J., \& Lynden-Bell, D. 1986, MNRAS, 221, 1023

Kirkpatrick, J. D., McGraw, J. T., Hess, T. R., Liebert, J., \& McCarthy, D. W. Jr. 1994, ApJS, 94, 749

Kirkpatrick, J. D., Barman, T. S., Burgasser, A. J., et al. 2006, ApJ, 639, 1120

Kroupa, P., Tout, C. A., \& Gilmore, G. 1993, MNRAS, 262, 545

Kuijken, K., \& Gilmore, G. 1989, MNRAS, 239, 605

Lee, T. A. 1968, ApJ, 152, 913

Lodieu, N., Pinfield, D. J., Leggett, S. K., et al. 2007a, MNRAS, 379, 1423

Lodieu, N., Dobbie, P. D., Deacon, N. R., et al. 2007b, MNRAS, 380, 712

Lodieu, N., Hambly, N. C., Jameson, R. F., \& Hodgkin, S. T. 2008, MNRAS, 383, 1385

Looper, D. L., Kirkpatrick, J. D., \& Burgasser, A. J. 2007, AJ, 134, 1162

Lucas, P. W., \& Roche, P. F. 2000, MNRAS, 314, 858

Lucas, P. W., Roche, P. F., Allard, F., \& Hauschildt, P. H. 2001, MNRAS, 326, 695

Luhman, K. L., Rieke, G. H., Young, E. T., et al. 2000, ApJ, 540, 1016

Majewski, S. R. 1993, ARA\&A, 31, 575

Martín, E. L., \& Zapatero Osorio, M. R. 2003, ApJ, 593, L113

Martín, E. L., Rebolo, R., \& Zapatero Osorio, M. R. 1996, ApJ, 469, 706

Martín, E. L., Basri, G., Zapatero Osorio, M. R., Rebolo, R., \& García López, R. J. 1998, ApJ, 507, L41

Martín, E. L., Delfosse, X., Basri, G., et al. 1999, AJ, 118, 2466

Martín, E. L., Brandner, W., Bouvier, J., et al. 2000, ApJ, 543, 299

Martín, E. L., Zapatero Osorio, M. R., Barrado y Navascués, D., Béjar, V. J. S., \& Rebolo, R. 2001, ApJ, 558, L117

McGovern, M. R., Kirkpatrick, J. D., McLean, I. S., et al. 2004, ApJ, 600, 1020

Metchev, S., Kirkpatrick, J. D., Berriman, G. B., \& Looper, D. 2008, ApJ, 676, 1281

Mohanty, S., Jayawardhana, R., \& Basri, G. 2005, ApJ, 626, 498

Moraux, E., Bouvier, J., \& Stauffer, J. R. 2001, A\&A, 367, 211

Moraux, E., Bouvier, J., Stauffer, J. R., \& Cuillandre, J.-C. 2003, A\&A, 400, 891

Moraux, E., Bouvier, J., Stauffer, J. R., Barrado y Navascués, D., \& Cuillandre, J.-C. 2007, A\&A, 471, 499
Muench, A. A., Lada, E. A., Lada, C. J., \& Alves, J. 2002, ApJ, 573, 366

Nakajima, T. 2005, IAC/TNG Workshop on Ultralow-mass star formation and evolution, La Palma, 2005 June 28-July 1, ed. E. L. Martín, \& A. Magazzù Paresce, F., de Marchi, G., \& Romaniello, M. 1995, ApJ, 440, 216

Phleps, S., Drepper, S., Meisenheimer, K., \& Fuchs, B. 2005, A\&A, 443, 929

Pinfield, D. J., Hodgkin, S. T., Jameson, R. F., Cossburn, M. R., \& von Hippel, T. 1997, MNRAS, 287, 180

Pinfield, D. J., Dobbie, P. D., Jameson, R. F., et al. 2003, MNRAS, 342, 1241

Pirzkal, N., Sahu, K. C., Burgasser, A., et al. 2005, ApJ, 622, 319

Preibisch, T., \& Zinnecker, H. 1999, AJ, 117, 2381

Prochaska, J. X., Naumov, S. O., Carney, B. W., McWilliam, A., \& Wolfe, A. M. 2000, AJ, 120, 2513

Rebolo, R., Martín, E. L., \& Magazzù, A. 1992, ApJ, 389, L83

Rebolo, R., Martín, E. L., Basri, G., Marcy, G. W., \& Zapatero Osorio, M. R. 1996, ApJ, 469, L53

Rees, M. J. 1976, MNRAS, 176, 483

Reid, M. J. 1993, ARA\&A, 31, 345

Reid, I. N., \& Hawley, S. L. 1999, AJ, 117, 343

Reid, I. N., Gizis, J. E., Cohen, J. G., et al. 1997, PASP, 109, 559

Rieke, G. H., \& Lebofsky, M. J. 1985, ApJ, 288, 618

Robin, A. C., Reylé, C., \& Crézé, M. 2000, A\&A, 359, 103

Ryan, Jr., R. E., Hathi, N. P., Cohen, S. H., \& Windhorst, R. A. 2005, ApJ, 631, L159

Scholz, A., \& Eislöffel, J. 2004, A\&A, 419, 249

Scholz, A., \& Jayawardhana, R. 2008, ApJ, 672, L49

Slesnick, C. L., Hillenbrand, L. A., \& Carpenter, J. M. 2004, ApJ, 610, 1045

Skrutskie, M. F., Cutri, R. M., Stiening, R., et al. 2006, AJ, 131, 1163

Stanway, E., Bremer, M. N., Lehnert, M. D., \& Eldridge, J. J. 2008, MNRAS, 384,348

Stauffer, J. R., Hamilton, D., \& Probst, R. G. 1994, AJ, 108, 155

Stauffer, J. R., Schultz, G., \& Kirkpatrick, J. D. 1998, ApJ, 499, L199

Stern, D., Kirkpatrick, J. D., Allen, L. E., et al. 2007, ApJ, 663, 677

Thi, W. F., van Dishoeck, E. F., Blake, G. A., et al. 2001, ApJ, 561, 1074

Tinney, C. G., Burgasser, A. J., \& Kirkpatrick, J. D. 2003, AJ, 126, 975

Tinney, C. G., Burgasser, A. J., Kirkpatrick, J. D., \& McElwain, M. W. 2005, AJ, 130,2326

Vrba, F. J., Henden, A. A., Luginbuhl, C. B., et al. 2004, AJ, 127, 2948

Warren, S. J., Hambly, N. C., Dye, S., et al. 2007, MNRAS, 375, 213

West, A. A., Hawley, S. L., Bochanski, J. J., et al. 2008, AJ, 135, 785

White, R. J., \& Basri, G. 2003, ApJ, 582, 1109

Zapatero Osorio, M. R., Rebolo, R., \& Martín, E. L. 1997, A\&A, 317, 164

Zapatero Osorio, M. R., Béjar, V. J. S., Rebolo, R., Martín, E. L., \& Basri, G. 1999, ApJ, 524, L11

Zapatero Osorio, M. R., Béjar, V. J. S., Martín, E. L., et al. 2000, Science, 290, 103

Zapatero Osorio, M. R., Béjar, V. J. S., Martín, E. L., Barrado y Navascués, D., \& Rebolo, R. 2002a, ApJ, 569, L99

Zapatero Osorio, M. R., Béjar, V. J. S., Martín, E. L., et al. 2002b, ApJ, 578, 536

Zapatero Osorio, M. R., Martín, E. L., Béjar, V. J. S., et al. 2007a, ApJ, 666, 1205

Zapatero Osorio, M. R., Caballero, J. A., Béjar, V. J. S., et al. 2007b, A\&A, 472, L9

Zapatero Osorio, M. R., Béjar, V. J. S., Bihain, G., et al. 2008, A\&A, 477, 895

Zheng, Z., Flynn, C., Gould, A., Bahcall, J. N., \& Salim, S. 2004, ApJ, 601, 500 